\title{
A solution based on Cellular Automaton for merging after toll
}

\author{
Wenjing Xiao \\ School of North China Electric Power University, Baoding 071000, China \\ 303208624@qq.com
}

\begin{abstract}
Based on Cellular Automaton (CA) and Monte Carlo method, this paper constructs a model to discuss the problem of merging after toll, and designs the shape, size, and merging pattern of the toll area. Firstly, the movement of the vehicle is broken down into three stages to establish the corresponding sub-models. Then we set up a series of indicators to analyze the toll plaza, such as Danger Index, Efficiency Index and cost. Fourth, we test our model via heavy traffic and autonomous (self-driving) vehicles. As the inflow probability of the vehicle increases, the vehicles slow down gradually but the speed is greater than zero. In summary, our model is powerful and reliable for simulating vehicles' merging after roll, coinciding with real statistics and suiting the dynamic and sophisticated features of merging process.
\end{abstract}

Keywords: Cellular Automaton, Monte Carlo method, merging after toll.

\section{Introduction}

With the continuous development of highways, highway traffic accident rate is also increased every year. Toll system is an important part of the highway. Currently, the "“barrier tolls" is one of the common highway toll charging methods. A barrier toll is a row of tollbooths placed across the highway, and there are usually (always) more tollbooths than there are incoming lanes of traffic. Therefore, when the car leaves the toll station, the traffic must be fan-shaped from the exit of the wider toll station into the less conventional lane of the lane

\section{The Models}

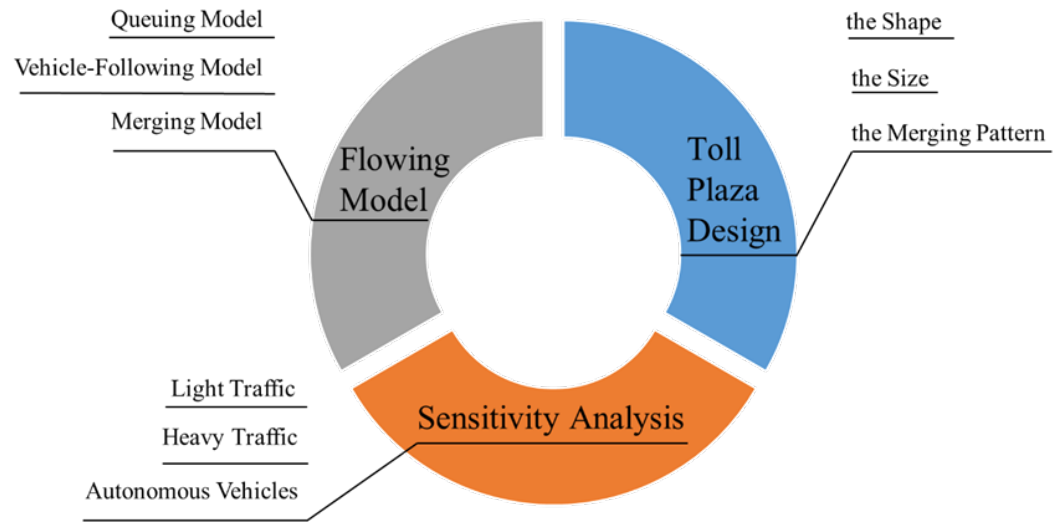

\subsection{Design of Cellular Automata ${ }^{[1]}$}

Fig1. Model structure diagram

Previously, a large number of traffic simulations were based on Cellular Automata (CA), indicating that the CA model is a feasible and effective method to emulate traffic flow. Space, time, and all state are discrete in Cellular Automata. Therefore, we apply Cellular Automata to solve our problem is reasonable.

We discuss the basic process of the CA model:

- Inflow Process: According to the inflow model that we will discuss in a moment, assign vehicles in the vehicle-generation area.

- Acceleration Process: If $V<V m$, a vehicle accelerates by $\Delta V$, and the new speed is $V^{\prime}=V+\Delta V$.

- Deceleration Process: If the distance between a vehicle and the vehicle ahead of it (Front bumper to front 
bumper distance, we call it the gap, and the gap is denoted by $G$ and its unit is cell. When there is no vehicle ahead, $G$ is set to $+\infty)$ is no more than $V$, the vehicle decelerates to $V^{\prime}=(G-1) / T$.

Moving Process: Vehicles move forward by $V^{\prime *} T$ cells only when $G>G_{s}\left(V^{\prime}\right)$. $G_{s}\left(V^{\prime}\right)$ is the minimum gap required for safety consideration, and is to be defined later.

\subsection{Flowing Model}

In order to describe the merge issues after the vehicle charges, we need to discuss the situation in the toll station service. Firstly, we establish the flow model, assuming that the random arrival of vehicles at the entrance of the expressway obey the Poisson probability distribution approximately. The state of the vehicle through the toll station as a vehicle combined initial state. Then we discuss the next step.

\section{Merging model}

\subsection{Pluggable gap theory}

The plug car clearance is a time interval that the driver can safely import or through the continuous flow. When more vehicles pass the toll plaza, the driver entering or crossing traffic must be judged from the potential conflict between the vehicle and the own vehicle to make a decision whether to enter or pass through.

\subsection{Pluggable gap theory}

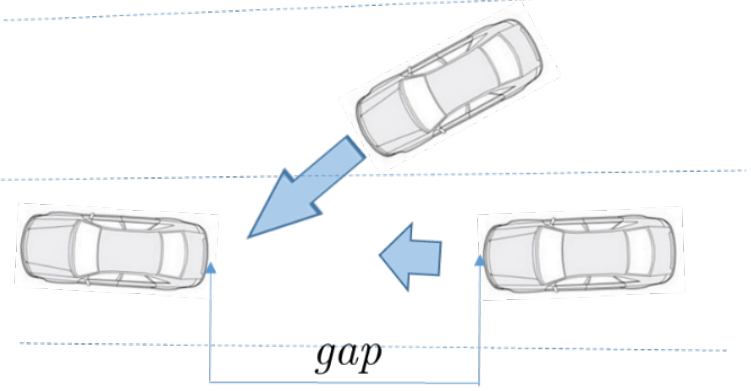

Fig2. Lane change diagram

The probability $P_{0}$ depends on the vehicle, the front vehicle $A$ and the rear vehicle $B$, and $C$ indicates the minimum value of the time slot into which the vehicle enters the vehicle, and $t_{f}$ indicates the vehicle $A$. In the follow-up time between vehicles $B$ 、 $C$,probability $P_{0}$ satisfies:

$$
P_{0}=\left\{\begin{array}{l}
P_{c}\left(1-0.9 e^{\left(t_{c}-t_{f}\right)}\right) \quad \text { if } t_{c}<t_{f} \\
0.1 \quad \text { if } t_{c} \geq t_{f}
\end{array}\right.
$$

Where $P_{c}$ The driver from the finish near the end of the resulting tendencies

\begin{tabular}{cccc}
\hline Distance from the exit & $\leq 100$ & $\leq 300$ & $\geq 300$ \\
\hline Pc & 0.95 & 0.9 & 0.85 \\
\hline
\end{tabular}

Table1. Vehicle merging tendency

The above equation reflects the greater the time difference, the assumption of the greater the possibility of the driver merging is reasonable. The probability distribution reflects the tendency well.

\subsection{The size of the existing charge area}

The gradual transition from the toll plaza to the conventional motor vehicle lane requires that the vehicle trav el smoothly.Therefore, the transition section should use the appropriate gradient rate. When the toll station an $\mathrm{d}$ the width of the road is determined, the longer the transition length, the smaller the gradient,the more natur al vehicles from the toll plaza into the conventional motor vehicle lanes.Linear transition should be smooth, $r$ 
ound, to avoid that the traffic path is too reluctant,at the same time we should pay attention to construction di fficulty.

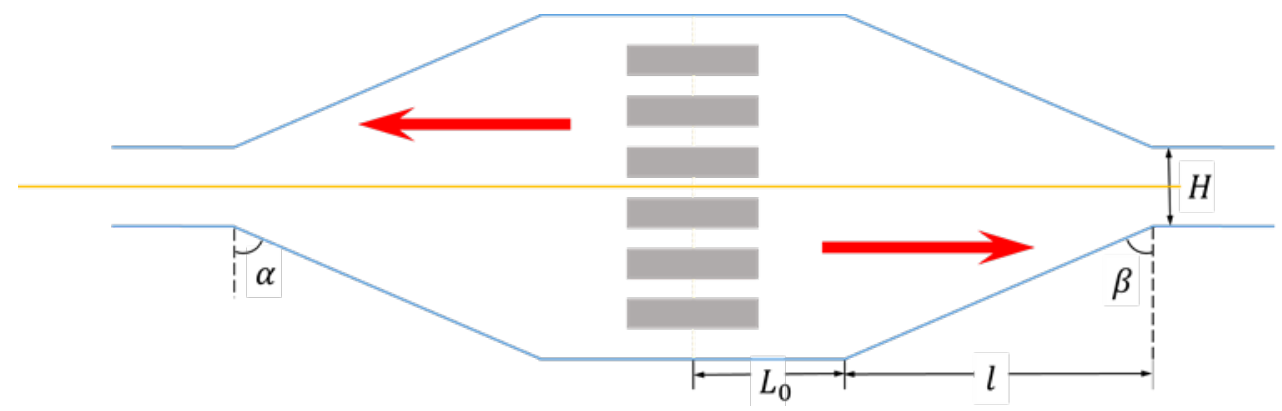

\section{The Design of Toll Plaza}

Fig3. Schematic diagram of toll plaza

\subsection{Performance measurement of toll plaza}

In order to compare the toll plaza, we set up some evaluation indexes from the four aspects of accident prevention, throughput, traffic efficiency and cost. The distance from the toll to the regular motor vehicle crossing is $M$; The number of vehicles in the distance is $n$ (vehicle/h) per unit time.

- accident prevention - risk factors

$$
d_{a}=\frac{\sum_{i=1, j=i+a}^{n-1}\left|d_{a i}-d_{a j}\right|}{n-1}
$$

passage efficiency - efficiency factor

$$
v=\frac{\sum_{i=1}^{n} v_{i}}{n}
$$

Traffic density: It refers to the number of vehicles distributed per unit length of lane per unit time and it indicates the concentration of the vehicle distribution:

$$
\rho=\frac{n}{M}
$$

- The cost of the existing toll plaza

The inflation rate used to be $1 \% \sim 3 \%$ each year. In order to simulate the real process, we set inflation rate at $1.5 \%$

Define the $C$ as the real median money. Thus we define the $\sigma$

$$
\sigma(t)=\sigma(t-1) \times(1+1.5 \%)
$$

The cost is calculated as:

$$
C=\left(C_{l}+C_{c}+C_{m}\right) \times \sigma(t) \times S^{\prime}
$$

- Performance analysis

According to the above calculation method, we take $L_{0}=50 m, \mathrm{D}=112 \mathrm{~m}, \alpha=72^{\circ}, \beta=72^{\circ}, B=8, L=3$. Toll stations takes artificial toll stations. The queuing theory parameters are $\lambda=0.2778$ (Vehicle/s), $\mu=0.0629$ (Vehicle/s) and $\Delta t=1 \mathrm{~s}$.Using cellular auto or simulation, the results of the indicators are as follows.

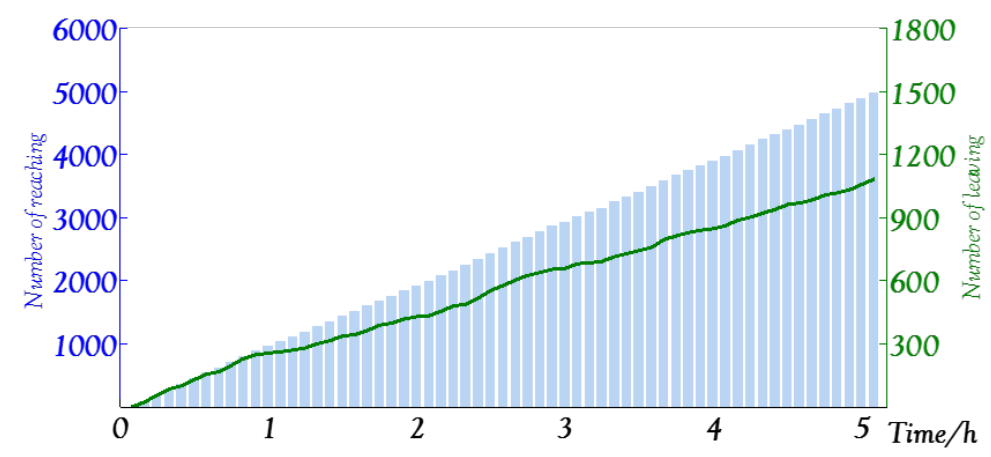

Fig4. The total number of vehicles with time 


\begin{tabular}{|c|c|c|c|c|c|c|}
\hline \multirow[b]{2}{*}{$\begin{array}{l}\text { Throughput } \\
\text { ( Vehicle/h) }\end{array}$} & \multicolumn{2}{|c|}{ Efficiency index } & \multicolumn{2}{|c|}{ Danger index } & \multirow[b]{2}{*}{ area (m2) } & \multirow[b]{2}{*}{ Cost } \\
\hline & $\begin{array}{c}\text { density } \\
\text { ( Vehicle/m2) }\end{array}$ & $\begin{array}{c}\text { average } \\
\text { velocity } \\
(\mathrm{m} / \mathrm{s})\end{array}$ & $\begin{array}{c}\text { average } \\
\text { vehicle } \\
\text { distance } \\
(\mathrm{m})\end{array}$ & $\begin{array}{l}\text { the number } \\
\text { of lane } \\
\text { changes per } \\
\text { hour }\end{array}$ & & \\
\hline 205.25 & 0.0065 & $\begin{array}{c}9.8 \\
9\end{array}$ & 58.13 & 1065.32 & 29462.97 & $\begin{array}{c}29462.97 \\
\text { C }\end{array}$ \\
\hline
\end{tabular}

Table2. The results of the indicators value

It can be seen that the number of vehicles entering the toll station and the toll station is gradually increasing with the increase of the time when the size of the toll plaza is $25000 \mathrm{~m}^{2}$,

\section{Conclusions}

With the continuous development of highway, expressway traffic accident rate is also increasing year by year. Toll system is an important part of the highway. In order to analyze the toll plaza, a certain amount of indicators have been set up. Indicators includes: Danger Index (containing average distance and average merging times) which is used to measure the better of accident prevention, Efficiency Index (containing average speed and density) defined to analyze the extent of traffic, as well as throughput and cost.

Then, the Cellular Automaton is taken to simulate traffic flow in a toll plaza design that may already be implemented. By changing the value of $\alpha$ and $\beta$, the simulation outcome of CA compared to the previous results as follows: the increasing of $\alpha$ improve the throughput and Efficiency Index but adds Danger Index as well, while the value of $\beta$ is positively related to the throughput and Efficiency Index, keeping the Danger Index simultaneously. In addition, both of them lightly contribute to the cost increase.

We also discuss the impact of the number of different tollbooths on merging after toll. According to the actual situation, assumption is that the average service rate of electronic toll collection booths is the fastest, the exact-change tollbooths second, conventional tollbooths last. Based simulation results, we find that the proper proportions of ETC or the exact-change tollbooths and conventional tollbooths should be from $1 / 3$ to $1 / 2$.Through multiple simulations, ensuring maximum safety and throughput, timely reducing the cost, the optimal design parameters of toll plaza.

Finally, we test our model via heavy traffic and autonomous (self-driving) vehicles.

As the probability of arrival of the vehicle increases, the flow of traffic gradually slow down.

Adding 5\% autonomous vehicles to the model, it is benefit to security, but not to throughput and efficiency.

\section{References}

[1] W. Strunk Jr., E.B. White, The Elements of Style, third ed., Macmillan, New York, 1979. Reference to a chapter in an edited book:

[2] J. van der Geer, J.A.J. Hanraads, R.A. Lupton, The art of writing a scientific article, J. Sci. Commun. 163 (2000) 51-59.

[3] G.R. Mettam, How to prepare an electronic version of your article, in: B.S. Jones, R.Z. Smith (Eds.), Introduction to the Electronic Age, E-Publishing Inc., New York, 1999, pp. 281-304.

[4] R.J. Ong, J.T. Dawley and P.G. Clem: submitted to Journal of Materials Research (2003)

[5] P.G. Clem, M. Rodriguez, J.A. Voigt and C.S. Ashley, U.S. Patent 6,231,666. (2001)

[6] Information on http://www.weld.labs.gov.cn 